\title{
A high repetition rate experimental setup for quantum non-linear optics with cold Rydberg atoms
}

\author{
Hannes Busche ${ }^{\mathrm{a}}$, Simon W. Ball, and Paul Huillery ${ }^{\mathrm{b}}$ \\ Joint Quantum Centre (JQC) Durham-Newcastle, Department of Physics, Durham \\ University, Durham, DH1 3LE, UK
}

Received 31 December 2015 / Received in final form 9 August 2016 Published online 19 December 2016

\begin{abstract}
Using electromagnetically induced transparency and photon storage, the strong dipolar interactions between Rydberg atoms and the resulting dipole blockade can be mapped onto light fields to realise optical non-linearities and interactions at the single photon level. We report on the realisation of an experimental apparatus designed to study interactions between single photons stored as Rydberg excitations in optically trapped microscopic ensembles of ultracold ${ }^{87} \mathrm{Rb}$ atoms. A pair of in-vacuum high numerical aperture lenses focus excitation and trapping beams down to $1 \mu \mathrm{m}$, well below the Rydberg blockade. Thanks to efficient magneto-optical trap (MOT) loading from an atomic beam generated by a $2 \mathrm{D} \mathrm{MOT}$ and the ability to recycle the microscopic ensembles more than 20000 times without significant atom loss, we achieve effective repetition rates exceeding $110 \mathrm{kHz}$ to obtain good photon counting statistics on reasonable time scales. To demonstrate the functionality of the setup, we present evidence of strong photon interactions including saturation of photon storage and the retrieval of non-classical light. Using in-vacuum antennae operating at up to $40 \mathrm{GHz}$, we perform microwave spectroscopy on photons stored as Rydberg excitations and observe an interaction induced change in lineshape depending on the number of stored photons.
\end{abstract}

\section{Introduction}

Non-linear optics with Rydberg atoms [1] has emerged as a viable approach to achieve strong effective interactions at the single photon level. Rydberg atoms exhibit strong and long-ranged dipolar interactions between each other $[2,3]$ which can be mapped onto optical signal fields using electromagnetically induced transparency (EIT) [4] coupling to a Rydberg state in a ladder scheme [5]. As the dipole blockade [6,7] turns a three level EIT system into an effective two-level system in the vicinity of a Rydberg

\footnotetext{
a e-mail: hannes.busche@durham.ac.uk

b e-mail: paul.huillery@durham.ac.uk
} 
excitation, strong optical non-linearities manifesting themselves in a suppression of transmission for increasing numbers of signal photons have been observed [8,9].

As a result of the non-linearity, highly non-classical states of light have been observed in optically thick media $[10,11]$ and by storing photons as long-lived collective Rydberg excitations in strongly confined volumes $[12,13]$. In a complementary experiment, sub-Poissonian excitation statistics have been observed in a non-linear Rydberg EIT medium using ion detection [14]. Attractive interactions between photons propagating through Rydberg media enabled the creation of two-photon bound states [15]. The change in transmission due to a nearby excitation can be used for spatial imaging of Rydberg atoms [16,17], e.g. to study the dynamics of Förster resonant energy transfer [18]. Similarly, a single stored photon has been used to control the transmission of light in a cold atomic ensemble realising all-optical switching [19] and single photon transistors [20,21], as well as the conditional application of a $\pi$-phase shift [22]. Thanks to the long life-times and the tunability of the interaction, photon storage in Rydberg states provides a convenient platform for these applications and future experiments in the context of optical quantum information processing.

Their high polarisabilty leads to strong interactions between Rydberg atoms, but also renders them sensitive to external fields. Consequently, the modified optical response of a Rydberg EIT medium [23] also provides a powerful tool to probe and image both DC electric [24-27] and microwave fields [28-32]. The application of external fields can also be used to enhance the effective photon interactions by introducing resonant dipole interactions: microwaves can directly couple to adjacent Rydberg states $[13,33]$, while the Stark shift resulting from a DC electric field can tune the interactions at a Förster resonance [34]. The application of a microwave pulse during storage is particularly interesting since it allows the storage and interaction processes to be decoupled by introducing interactions in a controlled manner. This could potentially be used to allow or prevent microwave rotations of a stored photonic qubit in a universal quantum gate [35] resembling similar schemes successfully implemented for neutral atoms [36].

Many of the aforementioned experiments and applications share the requirement to operate at high repetition rates. In quantum non-linear optics, the acquisition of large data sets is important for the analysis of photon statistics, while in metrology high repetition rates are required to enhance sensitivity. To create microscopic atomic clouds and address individual stored photons, optical resolution below the typical length scale of the blockade, i.e. a few $\mu \mathrm{m}$, is essential. Another important requirement is good control of external electromagnetic fields. In particular, we are interested in generating well-defined, reproducible microwave fields as a means to control interactions between Rydberg excitations. Similar overall requirements also apply for applications of Rydberg atoms outside quantum non-linear optics, such as single ion [37] and atom sources [38], and collectively encoded ensemble qubits [39,40].

Here we present details on a cold atom experiment fulfilling all these requirements. It is centred around a pair of high numerical aperture aspheric lenses, which provide an optical resolution of $1 \mu \mathrm{m}$ and are used to confine microscopic ${ }^{87} \mathrm{Rb}$ ensembles in tightly focussed optical dipole traps. These can be produced in less than $75 \mathrm{~ms}$ as a result of efficient loading of a magneto-optical trap (MOT) from an atomic beam generated by a two-dimensional magneto-optical trap (2D MOT) [41]. In addition, we recycle the trapped atoms for more than 20000 experimental shots of a few $\mu \mathrm{s}$ duration each before reloading the trap. By these means an effective repetition rate of $110 \mathrm{kHz}$ is achieved. Electric field control is provided by a set of electrodes, while microwave fields can be applied in a reproducible manner by three in-vacuum antennae. All of these have been integrated into the mount for the lenses. While not required for experiments presented in this paper, we also demonstrate the realisation of two side-by-side optical traps for future experiments in which we plan to study interactions between photons stored in spatially separated modes. 
We present a variety of experiments in which evidence of effective photon interactions is observed to demonstrate the capabilities of this setup. These include the suppression of EIT at high signal intensities, a saturation of the retrieved photon number in photon storage, and the retrieval of non-classical states of light. We also perform microwave spectroscopy on photons stored as Rydberg excitations and find a change in the observed lineshape due to the introduction of resonant dipole-dipole interactions into the system.

\section{Experimental setup}

In the following section, we present details of the experimental apparatus with a focus on achieving fast cycle-times and near-diffraction limited optical resolution on the order of $1 \mu \mathrm{m}$. The vacuum system is realised as a typical dual chamber setup: while typically used to achieve improved ultra-high vacuum conditions, i.e. to achieve long trap lifetimes for the production of degenerate quantum gases, we chose to implement a 2D MOT as a cold atomic beam source to achieve efficient MOT loading and thus minimise the overall cycle time. The most characteristic feature, however, is the inclusion of a pair of high numerical aperture aspherical lenses. They allow the production and addressing of microscopic ${ }^{87} \mathrm{Rb}$ ensembles in tightly focussed optical dipole traps. We show that at low signal intensities trap loss is sufficiently weak to allow several $10^{4}$ repetitions of typical experiments following a single MOT loading, which is a second crucial factor contributing to achieving high repetition rates. We also give a short description of measures taken to achieve good electric field control, as well as on the implementation of three antennae inside the vacuum chamber to drive microwave transitions between Rydberg states at up to $40 \mathrm{GHz}$. An overview of both the laser system used to couple to Rydberg states as well as the single photon detection setup is presented, and we demonstrate Rydberg EIT in a non-interacting regime.

\subsection{Two-dimensional magneto optical trap}

In order to achieve fast cycle times, a 2D MOT [41] was implemented as a switchable cold atomic beam source for efficient loading of the science MOT. The atomic beam is generated in a vacuum region with comparatively high $\mathrm{Rb}$ partial pressure (typically $10^{-7}$ to $10^{-8} \mathrm{mbar}$ ). This allows for fast loading speeds without compromising vacuum pressure in the main chamber. The atoms are laser cooled in two dimensions perpendicular to the desired beam axis, which is defined by the zero-line of an elongated quadrupole magnetic field, and thus magneto-optically confined. Atoms with lower velocity along the beam axis are more strongly confined due to undergoing a greater number of scattering events with the cooling light. A small aperture separating the $2 \mathrm{D}$ MOT and main science chamber preferentially permits cooler atoms with a low velocity along the beam axis to pass, ideally with the majority below the capture velocity of the MOT. This allows to maintain typical pressures below $10^{-10}$ mbar in the main science chamber. The 2D MOT setup realised and presented below is based on a design by Hofmann et al. [42].

The central element of the setup is a rectangular glass cell with inner dimensions of $25 \mathrm{~mm} \times 25 \mathrm{~mm} \times 150 \mathrm{~mm}$. The optical components and magnets required are mounted in a compact aluminium cage surrounding the cell as shown in Fig. 1. The glass cell, electrical feed-throughs for two alkali-metal dispensers, a differential pumping tube, $2 \mathrm{~L} / \mathrm{s}$ ion getter pump, and a vacuum valve, are all attached to a single rectangular stainless steel block (see Fig. 1(a)). The two dispensers (Alvatec Alvasource) each contain $250 \mathrm{mg}$ of $\mathrm{Rb}$ at natural isotopic abundance and are located 
(a)

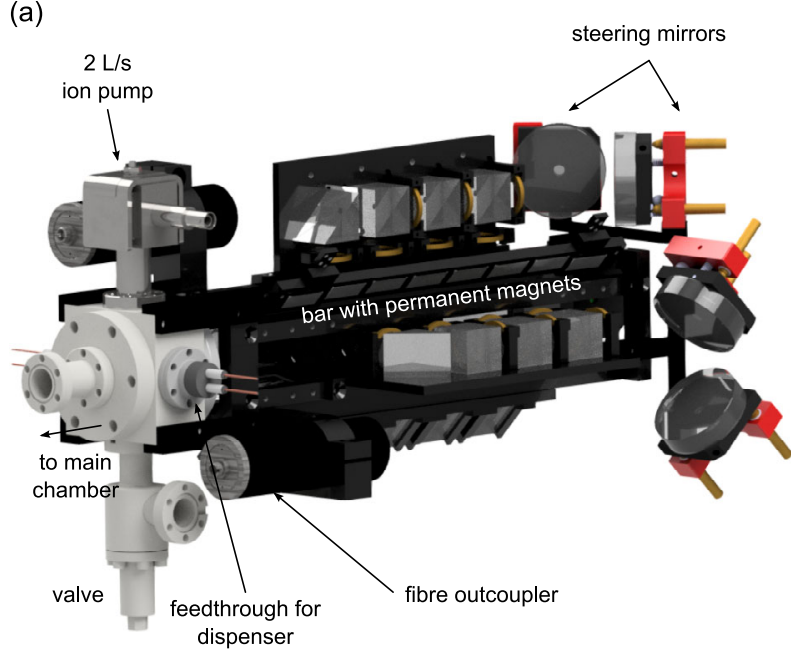

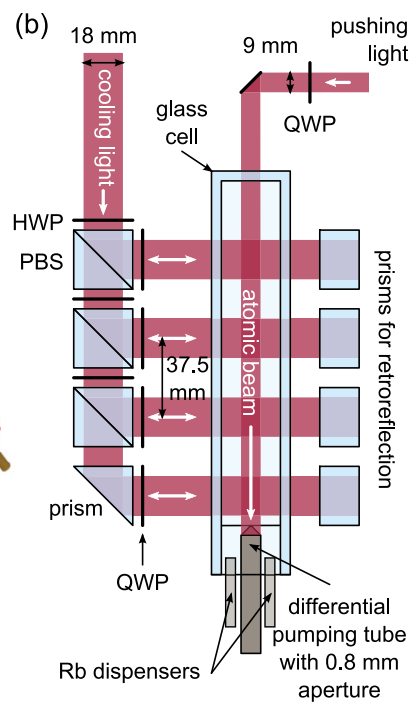

Fig. 1. 2D MOT setup. (a) Overview. All optics and magnets required are mounted on a cage structure surrounding a ultra-high vacuum glass cell. Dispenser feed-throughs, valves, an ion pump, and the cell attach to a square metal block. (b) Optical setup. The cooling light is divided into circularly polarised beams for four separate cooling regions using a combination of polarising beam splitters (PBS) and half and quarter wave plates (HWP/QWP). A circularly polarised pushing beam is co-propagating on the atomic beam axis. The beam sizes given correspond to the respective $1 / e^{2}$ diameters.

inside the tubing leading to the rectangular part of the cell. Vacuum inside the cell is maintained through a $149 \mathrm{~mm}$ long differential pumping tube with an inner diameter of $6 \mathrm{~mm}$ and a $0.8 \mathrm{~mm}$ diameter aperture at the 2D MOT end to velocity select the slow atoms in the atomic beam. The aperture size was chosen based on other designs [42] to allow for a high atomic flux on the order of $10^{9} \mathrm{~s}^{-1}$ to pass while at the same time preventing undesired leakage of atoms into the main science chamber and maintaining sufficiently good vacuum on the 2D MOT side without a large ion pump. To permit independent vacuum breaks, a gate valve is placed between the $2 \mathrm{D}$ MOT section and the main science chamber.

The optical setup is presented in Fig. 1(b). The $780 \mathrm{~nm}$ cooling $\left(5 S_{1 / 2}, F=2\right.$ to $\left.5 P_{3 / 2}, F^{\prime}=3\right)$ and repumping light $\left(5 S_{1 / 2}, F=1\right.$ to $\left.5 P_{3 / 2}, F^{\prime}=2\right)$ is delivered to the 2D MOT via two optical fibres and is split into four separate cooling regions with a distance of $37.5 \mathrm{~mm}$ between centres using a combination of polarising beamsplitter cubes (PBS) and wave plates. The circularly polarised cooling beams have a $1 / e^{2}$-diameter of $18 \mathrm{~mm}$ and are retro-reflected by rectangular prisms [43] thus maintaining the helicity of the polarisation without the use of additional quarter waveplates (QWP). A red-detuned pushing beam with an $1 / e^{2}$-diameter of $9 \mathrm{~mm}$ is co-propagating on the beam axis in order to apply a force on atoms which are initially moving in the wrong direction such that their direction is changed to contribute additional flux. The elongated quadrupole magnetic field is created using permanent magnets [44] and has a gradient of $\approx 16 \mathrm{G} / \mathrm{cm}$. In addition, two pairs of rectangular Helmholtz coils (not shown) are attached to the outside of the cage in order to compensate stray magnetic fields, i.e. the earth magnetic field, which cause a shift of the atomic beam axis with respect to the aperture.

In the main science chamber, the atoms are captured in a standard three dimensional MOT. The three retro-reflected MOT beams have a $1 / e^{2}$-diameter of $9 \mathrm{~mm}$, 


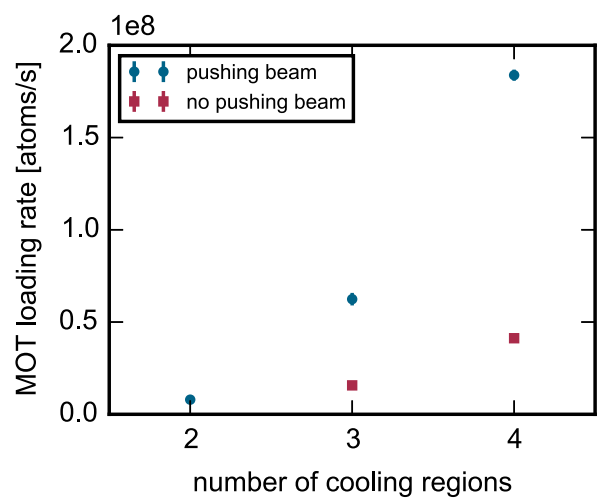

Fig. 2. Influence of cooling volume size on $2 \mathrm{D}$ MOT performance. The MOT loading rate is shown as different numbers of cooling regions are used comparing the performance with (blue circles) and without (red squares) use of the pushing beam.

though for the beams in the radial plane the diameter is truncated at $4 \mathrm{~mm}$ in one dimension to avoid clipping and reflections on the closely spaced in-vacuum aspheric lenses. During loading from the 2D MOT, we typically operate the MOT at $20 \mathrm{~mW}$ total cooling power and $-12 \mathrm{MHz}$ red detuning, $0.3 \mathrm{~mW}$ repumping power, and an axial magnetic field gradient of $34 \mathrm{G} / \mathrm{cm}$. The $2 \mathrm{D}$ MOT is operated at $140 \mathrm{~mW}$ total cooling power and $-8.5 \mathrm{MHz}$ red detuning, $3 \mathrm{~mW}$ repumping power, and a power of $20 \mathrm{~mW}$ and detuning of $-35 \mathrm{MHz}$ for the pushing beam. We also note that the performance is strongly dependent on the $\mathrm{Rb}$ vapour pressure, which can be controlled via the current applied to the dispensers, though we refrain from presenting a quantitative measurement here, as we have no direct way of measuring the $\mathrm{Rb}$ pressure inside the glass cell and the optimal dispenser current depends on the individual dispenser used.

Figure 2 shows the performance of the MOT loading depending on the size of the 2D MOT cooling volume. To reduce the cooling volume, cooling regions were blocked one-by-one starting with the one furthest away from the aperture. We find a significant reduction in the loading rate as the cooling volume is decreased. We presume that a long cooling volume is particularly important in our setup. Due to the small size of our MOT beams, we should strongly benefit from improved collimation of the atom beam as a result of the additional transverse cooling. We also compare the loading rates with and without application of the pushing beam and find that it enhances the MOT loading rate by a factor of $\approx 4.5$ when all four cooling regions are used.

When all parameters are optimal, the MOT atom number saturates at $(2.5 \pm 0.1)$. $10^{7}$ in approximately $150 \mathrm{~ms}$. Since comparable setups with larger MOT beams can achieve higher loading rates and atom numbers [41-43,45], we consider the limited size of our MOT beams and the resulting lower capture velocity to be the dominant constraint on loading performance and final atom number.

\subsection{In-vacuum aspheric lenses}

We aim to create cold atomic ensembles with dimensions of just a few $\mu \mathrm{m}$ in tightly focused dipole traps, and to individually address photons stored as Rydberg excitations. To achieve this, we must focus the trap light at $910 \mathrm{~nm}$ and the signal light at $780 \mathrm{~nm}$ well below the typical extent of the dipole blockade between Rydberg atoms. This requires near diffraction limited optical resolution on the order of $1 \mu \mathrm{m}$. This is achieved using a pair of high numerical aperture aspheric lenses with an effective 
(a)

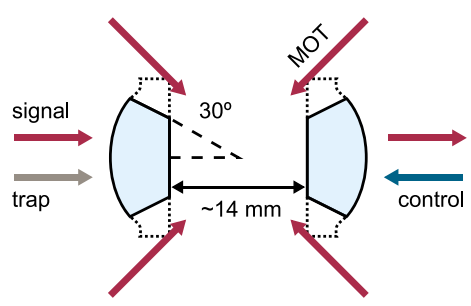

(b)

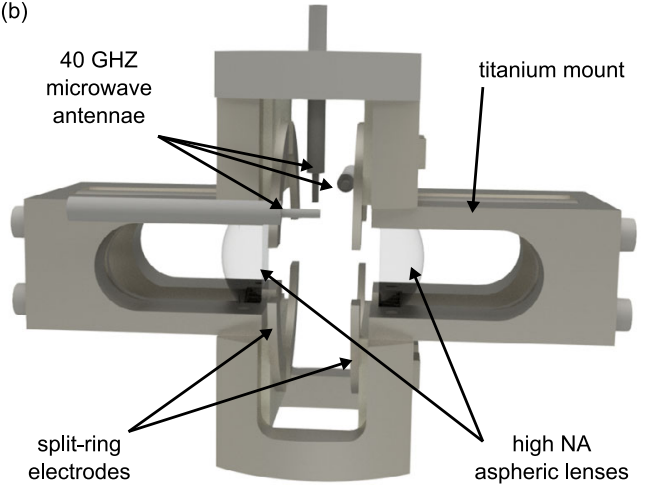

Fig. 3. In-vacuum lens assembly. The mount for the high NA aspheric lenses (effective focal length $\approx 10.3 \mathrm{~mm}$ ) was milled from a single titanium block. A set of split ring electrodes as well as three simple microwave antennae rated up to $40 \mathrm{GHz}$ made from open ended SMA$\mathrm{K}$ cable were incorporated into the assembly. Macor holders for the antennae and a pair of anti-Helmholtz coils generating the magnetic gradient field the MOT are not shown for simplicity. All titanium and Macor surfaces have been coated with a thin platinum layer to avoid the built-up of patch charges.

focal length of $f=10.3 \mathrm{~mm}$ and working distance of $7 \mathrm{~mm}$ placed inside the vacuum chamber. The exact distance between the lenses was optimised such that an initially collimated beam of signal light at $780 \mathrm{~nm}$, or signal light retrieved in the same spatial mode after storage, is re-collimated by the output lens. Consequently, beams of different wavelengths - i.e. the dipole trap light at $910 \mathrm{~nm}$ and control light at $480 \mathrm{~nm}-$ must have their convergence adjusted to co-locate their foci with the signal light. In order to provide sufficient optical access despite their close spacing of $\approx 14 \mathrm{~mm}$, i.e. to allow the MOT beams to intersect at the standard angle of 90 degrees, the sides of the lenses have been cut at an angle of 30 degrees (see Fig. 3(a)).

For reference measurements of beam sizes and positions as well as to assist coarse alignment of the signal and trap beams, an identical reference lens is placed outside the vacuum. From knife edge measurements carried out with a piezo-driven translation stage with sub- $\mu \mathrm{m}$ resolution, we measure a $1 / e^{2}$-waist of $1.0 \pm 0.1 \mu \mathrm{m}$ for an incoming collimated signal beam with radius $3.75 \mathrm{~mm}$, which is close to the expected diffraction limited spot size $(\approx 0.9 \mu \mathrm{m})$ of the cut lens. However, we note that the actual uncertainty on the waist is most likely larger than the standard errors stated due to unevenness of the knife edge.

Due to the sensitivity of Rydberg atoms to electrical fields, particular care must be taken to avoid the build up of patch charges caused by deposition of atoms on ungrounded or dielectric surfaces, which can lead to time-varying background fields [24]. Consequently, the lens surfaces facing the atoms have been coated with a conductive layer of indium tin oxide (ITO, sheet resistance $150 \Omega /$ sq) which is in electrical contact with the grounded lens mount. This layer substantially decreases the transmission of each lens, to $84 \%(82 \%)$ at $780 \mathrm{~nm}(910 \mathrm{~nm})$.

The lenses are held in place by a mount manufactured from a single block of titanium (Fig. 3(b)) which was chosen over stainless steel for its magnetic properties and lower thermal expansion during bake-out. Titanium, like aluminium, rapidly forms a dielectric oxide layer on its surface during contact with air. As with the lenses, to avoid patch charge build up, a $50 \mathrm{~nm}$ layer of platinum was deposited on all surfaces in line of sight of the atoms. In addition to the lenses, a set of eight electrodes, mounted in Macor blocks, and three microwave antennae are also incorporated into the lens 
(a)

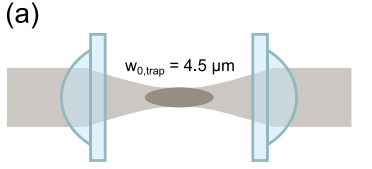

(b)

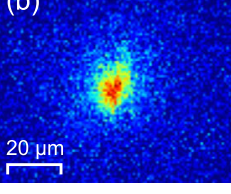

(c)

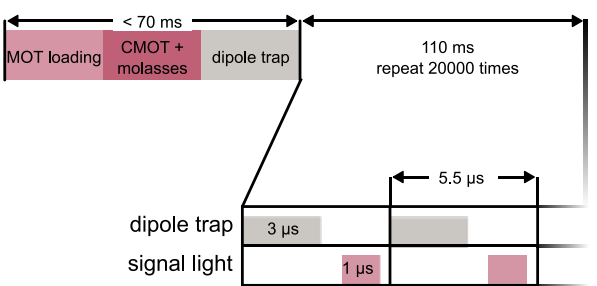

(d)

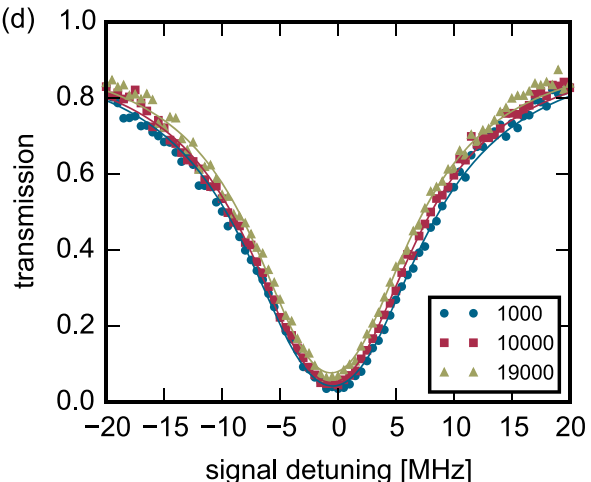

Fig. 4. Microscopic dipole trap. (a) Optical layout and dimensions. (b) Fluorescence image of the trap taken at 39 fold magnification averaged over 800 shots. The cloud size exceeds the trap beam dimensions due to expansion during the exposure time of $20 \mu \mathrm{s}$. (c) Typical experimental sequence. Following loading, the MOT is compressed and grey molasses is applied to increase the density and reduce the temperature of the atoms. Then, the dipole trap is switched on and off between each experimental shot, thus allowing to repeat an experiment more than 20000 times. (d) Transmission of signal light through the dipole trap after 1000 (blue circles), 10000 (red squares), and 19000 (yellow triangles) repetitions. For a weak signal beam of $3.1 \pm 0.2$ photons, no reduction in the optical depth of the trap is observed as the cloud is probed repeatedly.

mount assembly and are described further below. As Macor is a ceramic, the same platinum coatings have been applied as on the titanium surfaces.

\subsection{Microscopic dipole trap}

We utilise an attractive microscopic optical dipole trap [46] to create cold atom ensembles confined to the length scale of the Rydberg blockade - i.e a few $\mu \mathrm{m}$. This is created by a single beam, far red-detuned from the $\mathrm{Rb} D 1$ and $D 2$ lines, tightly focused by the in-vacuum aspheric lenses (see Fig. 4(a)). The trap light is provided by a homebuilt amplified diode laser with a wavelength of $910 \mathrm{~nm}$. A polarisation maintaining single mode (PM) optical fibre delivers the light to the experiment, which is then coaxially overlapped with the signal light. This ensures that both beams take the same path through the aspheres and the foci of each will be located on the same axis without further relative alignment. Since the focal length of the aspheric lens is wavelength dependent, the outcoupling lens for the trap light fibre is placed in a translation mount to match the longitudinal position of the trap focus to that of the signal light by adjusting the trapping beam convergence.

In order to approximately match the diameter of the atom cloud to that of the signal beam, we choose a trapping beam waist of $4.5 \pm 0.5 \mu \mathrm{m}$ at the focus which is confirmed by a measurement using the reference lens placed outside the vacuum. At a typical beam power of $50 \mathrm{~mW}$ inside the chamber, this results in a calculated axial trapping frequency of $\approx 0.65 \mathrm{kHz}$ and a radial frequency of $\approx 13 \mathrm{kHz}$, the calculated trap depth is $428 \mu \mathrm{K}$. A fluorescence image of the trapped atoms is shown in Fig. 4(b). Here, the atoms are imaged through the second in-vacuum asphere and a $400 \mathrm{~mm}$ lens outside the vacuum at $\approx 39$ fold magnification. We note that the size of the cloud in the image exceeds the trap waist due to ballistic expansion during the exposure time of $20 \mu$ s and parts of the elongated cloud being outside the depth of field of the asphere. In addition, the expansion is enhanced due to heating by the imaging light. 
Due to these imperfections in the imaging, we refrain from inferring parameters such as cloud dimensions, atom number, or temperature. Instead, we typically characterise the trap via absorption of signal light which can be measured in less than $1 \mu \mathrm{s}$ (see below). We roughly estimate a radius of $\sigma_{r}=2.5 \mu \mathrm{m}$ for the atom cloud as we observe strongest absorption of signal light for a beam with a similar waist. Axially, we estimate $\sigma_{z}=40 \mu \mathrm{m}$ by scaling the trapping beam Rayleigh range with the ratio of the radial size compared to the beam waist. To reduce noise, the image presented was averaged over 800 shots.

A typical experimental sequence is shown in Fig. 4(c). The trap is switched on at full power from the start of the MOT loading which takes less than $50 \mathrm{~ms}$ as we do not require a fully loaded MOT to achieve a fully loaded dipole trap due to the small dimensions of the latter. To increase the density and reduce the temperature of the atoms, the MOT is compressed by linearly increasing the axial magnetic field gradient to $50 \mathrm{G} / \mathrm{cm}$ and the MOT red-detuning to $-25 \mathrm{MHz}$ over a period of $12 \mathrm{~ms}$. The atoms are then subject to a far detuned dark molasses for $0.5 \mathrm{~ms}$ (cooling power $7 \mathrm{~mW}$, detuning $-30 \mathrm{MHz}$ ) during which the magnetic field gradient is ramped to zero, to reach temperatures on the order of $50 \mu \mathrm{K}$. The atoms are held in the trap for $10 \mathrm{~ms}$ to allow any atoms trapped in the MOT, but not in the dipole trap, to move out of the region of interest. During this hold time, the MOT detuning is ramped to a blue detuning of $+10 \mathrm{MHz}$ to accelerate the process by pushing the atoms away. The atoms in the dipole trap remain unaffected, as they still experience a red detuning due to the light shift induced by the trap.

Despite the fast MOT loading achieved, the duration still exceeds the few $\mu s$ necessary for a typical storage-retrieval sequence by more than four orders of magnitude. To maximise the overall repetition rate, it is therefore crucial to perform as many sequences per MOT load as possible. This is limited by the rate at which atoms are lost from the trap region. Due to the light shift induced by the trapping beam, performing experiments while keeping the trap on is not practical. Instead, we pulse the trap such that it is off during an experiment and turned on again after each shot to ensure the atoms remain confined. A typical sequence is shown again in Fig. 4(c) where during a $5.5 \mu \mathrm{s}$ cycle the trap is on for $3 \mu \mathrm{s}$ and then switched off for a $2.5 \mu \mathrm{s}$ window to perform experiments. As experiments are repeated, we identify two important loss mechanisms: firstly, atoms may be lost as they move out of the trap region while the trap is turned off; secondly, the absorption of resonant signal light can induce light assisted collisions. In Fig. 4(d), we show absorption spectra measured after 1000, 10000, and 19000 experimental repetitions on the same atom cloud for 1000 subsequent shots. The atom cloud is probed on the $5 S_{1 / 2}, F=2, m_{F}=2$ to $5 P_{3 / 2}, F^{\prime}=3, m_{F^{\prime}}=3$ transition by a signal pulse containing a mean of 3.1 photons for a duration of $1 \mu \mathrm{s}$. Each datapoint is averaged over 8000 shots in total. No significant reduction in optical depth, typically on the order of 2.5 , is observed for up to 20000 repetitions. This implies that in the limit of weak signal pulses there is no significant heating or loss due to the signal light, i.e. in the case of a single signal photon on average which is of particular interest for applications of Rydberg quantum optics in quantum technology. Adding up $72.5 \mathrm{~ms}$ to prepare an microscopic atom cloud and $20000 \cdot 5.5 \mu \mathrm{s}=110 \mathrm{~ms}$ during which experiments are performed, we claim an "effective" repetition rate of $20000 / 182.5 \mathrm{~ms} \approx 110 \mathrm{kHz}$. The exact number of repetitions that is realistic without significant trap loss depends on the experimental conditions, i.e. the number of signal photons which determines the rate at which the cloud is heated and the initial temperature of the atom cloud. Working in the weaker single photon regime for example, we can typically carry out around 30000 repetitions before observing a strong reduction in optical depth.

A future alternative to pulsing the trap would be the use of trapping light at a magic wavelength where both ground and Rydberg state experience the same light shift [47-49]. The trap however, would suffer from the disadvantage that the light 

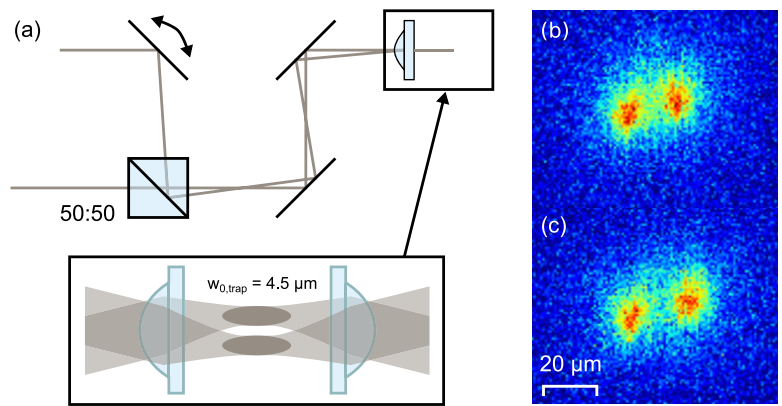

Fig. 5. Realisation of side-by-side optical dipole traps. (a) Optical layout. The distance between the trap can be varied by adjusting the angle at which the beams are incident on the aspheric lens. (b, c) Fluorescence images of the traps at different distances.

shift is state dependent, and the trapping potential repulsive for $n P$-states, thus not permitting the use of different Rydberg states at the same time. More suitable for our purposes might be the use of blue detuned, non-harmonic traps that allow confinement at an intensity minimum, in particular at zero intensity such that the atoms experience no light shift. Potentially suitable approaches include hollow beam traps [50] or box potentials formed by light sheets [51].

\subsubsection{Multiple traps}

To allow future investigation of interactions between photons propagating through or stored in spatially separated modes, we create two microscopic dipole traps side-byside. The distance between the traps can be adjusted by changing the incident angle of one of the trap beams on the aspherical lens, in order to translate the waist position in the focal plane (see Fig. 5(a)). In analogy to the single trap setup, signal and trap light for both sites are overlapped beforehand to decouple their relative alignment from alignment through the lens. A mismatch in the focal planes of the trapping beams is avoided since both originate from the same fibre output, behind which they are split into two separate beams having the same path length, before they are recombined again on a non-polarising beamsplitter (NPBS). A NPBS was preferred as it allows to adjust the polarisation of the signal light independently at each site, despite rejecting $50 \%$ of the trap light. Fluorescence images of the two side-by-side traps are shown in Figs. 5(b, c) for different separations, taken for similar parameters as for the single trap in Fig. 4(b). Again, the atom clouds are larger than their actual size prior to release from the trap due to ballistic expansion and fluorescent induced heating during the exposure time of $20 \mu \mathrm{s}$ and the images are averaged over 800 shots.

As scaling the number of traps by adding additional beams quickly becomes infeasible, more sophisticated methods may become of interest to create larger trap arrays in the future, i.e. using spatial light modulators to holographically generate freely configurable arrays similar to Nogrette et al. [52] who use the same type of lenses as in our setup except that their lenses remain uncut, or blue detuned arrays of Gaussian beams [53].

\subsection{Electric and microwave fields}

Since the high polarisability of Rydberg atoms renders them sensitive to external electric fields, good cancellation of stray fields is required, in particular at high 
principal quantum numbers. At the same time the controlled application of electric fields provides a powerful means to tune interactions between Rydberg excitations using Förster resonances. Active control is achieved using a set of eight electrodes which are configured in two rings surrounding the lens axis [54] (see Fig. 3). Each ring is cut into four segments, thus allowing the application of DC fields in arbitrary directions. The microscopic dimensions of our cold atom cloud do not require high field homogeneity over a large region, and consequently the electrode geometry has been optimised for unobstructed optical access.

Microwave fields can be used to drive transitions between adjacent Rydberg states. In particular, they allow the transfer of Rydberg excitations into states with angular momentum $\Delta L= \pm 1$ introducing long-ranged resonant dipole-dipole interactions. This enables both decoupling the photon storage from the interaction process, and also the study of spin or non-radiative energy exchange processes [55]. We opted to place three antennae inside the vacuum system, one in each spatial direction, rather than rely on an external antenna or horn (see Fig. 3(b)). Internal antennae close to the atoms are advantageous as the intensity profile and polarisation of the emitted microwave fields are influenced less by nearby metal objects or changes in an antenna's position. Thanks to the large transition strengths between adjacent Rydberg states, only low intensities are needed. Hence, the antennae are realised as compact, albeit inefficient, stub antennae using open ended coax cables with the core conductor protruding approximately $4 \mathrm{~mm}$ beyond the shielding. This realises a quarter-wave stub for frequencies around $18.7 \mathrm{GHz}$. The cables and vacuum feedthroughs $(2.92 \mathrm{~mm}$ SMA-K connectors) are rated up to a frequency of $40 \mathrm{GHz}$ setting a lower limit of $n=48$ for the principal quantum number for driving $n S_{1 / 2}$ to $(n-1) P_{1 / 2}$ transitions, though the signal generator (Anritsu MG3696A) is able to operate at frequencies up to $65 \mathrm{GHz}$.

\subsection{Rydberg excitation and detection}

A level scheme of the ${ }^{87} \mathrm{Rb}$ states we typically use, for both photon storage and $\mathrm{cw}$ Rydberg-EIT experiments is shown in Fig. 6(a). The signal light at $780 \mathrm{~nm}$ is resonant with the $5 S_{1 / 2}, F=2$ to $5 P_{3 / 2}, F^{\prime}=3$ transition on the $D 2$ line, while the control light couples the intermediate $5 P_{3 / 2}, F^{\prime}=3$ state to a Rydberg state $n S_{1 / 2}$. The principal quantum number $n$ is varied according to the type of experiment carried out. Even though they are less strongly interacting, we usually prefer $S$ - to $D$-states due to their simpler level structure, lower sensitivity to stray electric fields, and the isotropic and repulsive character of the interaction at high $n$.

Figure 6(b) shows a simplified schematic of the laser systems used to generate the signal and control light. The signal light is derived from the same diode laser (Toptica DL pro) which also provides the light needed for laser cooling and is frequency stabilised $140 \mathrm{MHz}$ below the $5 S_{1 / 2}, F=2$ to $5 P_{3 / 2}, F^{\prime}=3$ transition by modulation transfer spectroscopy [56]. Before being transferred to the experiment through a PM fibre, the light is tightly focussed into an acousto-optic modulator in a double pass configuration to allow frequency scans around the signal transition and fast switching with rise times around $20 \mathrm{~ns}$. The control light at $480 \mathrm{~nm}$ is produced by a frequency doubled amplified diode laser (Toptica TA SHG-110). It is frequency stabilised to the transition between the intermediate and Rydberg state using EIT spectroscopy [57]. As $n$ increases, the transition dipole moment between the intermediate and Rydberg states falls rapidly, limiting the upper state to which the control light can be stabilised. We therefore send the entire output of the laser through a Rb vapour cell used for the EIT lock, rather than splitting a fraction off for frequency stabilisation. With our current setup, this places a practical limit at around $n=90$. After the 
(a)

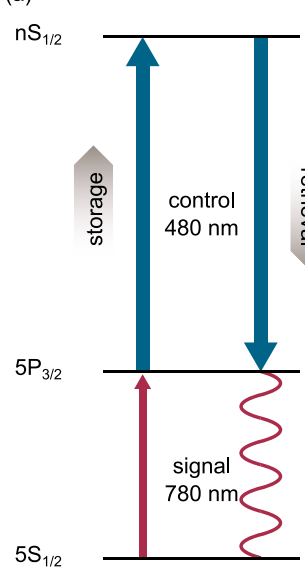

(b) $480 \mathrm{~nm}$
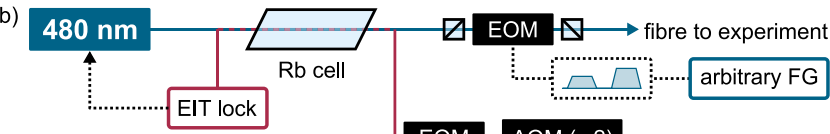

$\mathrm{EOM}-\mathrm{AOM}(\times 2)$

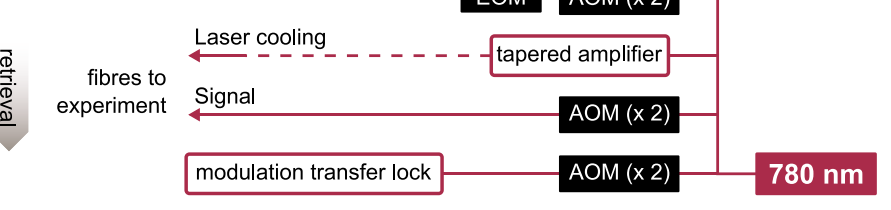

(c)

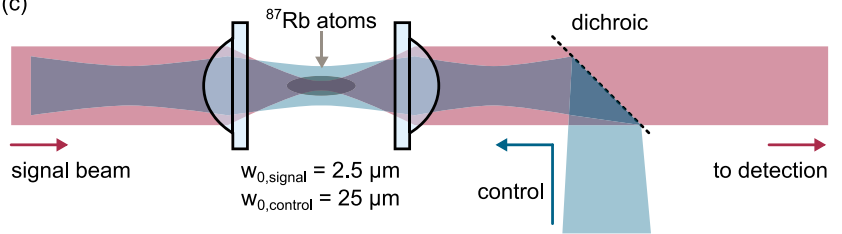

Fig. 6. Rydberg excitation. (a) Overview of atomic states and transitions used for EIT and photon storage in ${ }^{87} \mathrm{Rb}$. (b) Overview of the laser system. The signal light originates from the same diode laser as the light for the 2D MOT and MOT which is stabilised using modulation transfer spectroscopy. The control light is supplied by a frequency doubled diode laser stabilised to an EIT spectrum generated by overlapping signal and control light in a vapour cell. The light is switched using a polarisation switching EOM placed between two crossed Glan-Taylor polarisers. An arbitrary function generator is used to control the pulse shape. (c) Optical layout for EIT and photon storage. The signal and control beams are counter-propagating and focussed onto the microscopic Rb cloud by the in-vacuum aspheric lenses to waists of approx. $w_{S}=2.5 \mu \mathrm{m}$ and $w_{C}=25 \mu \mathrm{m}$, respectively.

cell, the light is switched by a polarisation switching electro-optic modulator (EOM) placed between two crossed Glan-Taylor polarisers. Typically the polarisation extinction ratio achieved by the EOM is slightly better than $20 \mathrm{~dB}$ such that some residual light is transmitted through the second polariser. However, we find that this leakage occurs in a slightly displaced spatial mode. In combination with the PM fibre used to transfer the light to the experiment, we therefore can achieve extinction ratios exceeding $46 \mathrm{~dB}$ at the fibre output before the science chamber.

Inside the science chamber, signal and control light counter-propagate with opposing circular polarisation and are strongly focussed onto the microscopic atom cloud by the aspheric lenses (see Fig. 6(c)). The signal beam is typically focussed to a waist of $\approx 2.5 \mu \mathrm{m}$, rather than the $1.0 \mu \mathrm{m}$ achieved above, matching its size to that of the atom cloud to maximise optical depth. The control light is focussed to a significantly larger waist of $\approx 25 \mu \mathrm{m}$ to achieve a reasonably large beam profile for future experiments involving spatially separated traps. To achieve this in spite of the short focal length of the lenses, we focus the control beam shortly before the lens. As the actual waist size is highly sensitive on the exact position of this focus, we refrain from inferring the control Rabi frequency from intensity measurements and rather determine it by fitting to transmission data in EIT/Autler-Townes spectra.

\subsubsection{Single photon detection}

To detect signal photons retrieved after storage or transmitted through the atom cloud in an EIT experiment, the signal beam is re-collimated using the second aspheric lens and reduced in beam diameter by a $4: 1$ telescope to allow efficient coupling into a PM single mode fibre (see Fig. 7). The single mode fibre serves as a spatial filter 


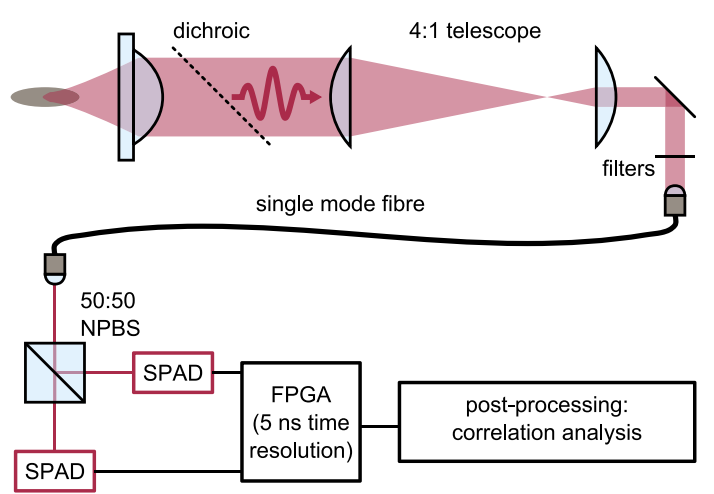

Fig. 7. Detection of signal light. Following re-collimation by the second in-vacuum lens the beam diameter is reduced for efficient coupling into a single mode fibre. Laser line filters before the fibre block the $910 \mathrm{~nm}$ trap light. After the fibre the light is detected by two single photon counters (SPADs) in a Hanbury-Brown-Twiss configuration. The timings of all detection events are recorded independently on a FPGA with $5 \mathrm{~ns}$ resolution. Any analysis of photon correlations is performed in post-processing.

separating signal light retrieved into the same spatial mode as the incoming beam from light randomly emitted from the atomic cloud, e.g. by spontaneous emission due to decay of dephased Rydberg excitations. These may be collected by the lens as it covers about $10 \%$ of the solid angle due to its high numerical aperture. A pair of laser line filters is placed before the fibre to block the dipole trap light and back reflections of the control beam.

Behind the fibre, two single photon avalanche detectors (SPADs, models PerkinElmer/Excelitas SPCM-AQRH-14-FC, customised to provide a TTL-compatible output pulse) and a non-polarising beamsplitter are arranged to form a HanburyBrown-Twiss interferometer allowing the analysis of photon pair correlations. The overall detection efficiencies are $\eta_{A}=11.6 \pm 0.9 \%$ and $\eta_{B}=8.5 \pm 0.7 \%$ respectively including all losses from the atoms to the detector. Main factors contributing to the finite detection efficiency are reflections from the non-index matched ITO coating on the collection lens ( $16 \%$ loss), the linewidth filters ( $33 \%$ loss), imperfect coupling into the single-mode fibre ( $24 \%$ loss), and the quantum efficiency of the SPADs ( $40 \%$ loss). Detected photons are signalled by a TTL-compatible voltage pulse generated by the SPAD and transmitted to digital inputs of an FPGA. The arrival times of the detected photons are recorded independently for each SPAD with a time resolution of $5 \mathrm{~ns}$. Consequently, any correlations between the arrival times of the photons can be analysed in post-processing, rather than using a start-stop method where only the timings between clicks of the different detectors are recorded.

Since the setup is dedicated to experiments in Rydberg non-linear optics, we decided to focus entirely on optical means of detection and no ion detection of Rydberg atoms using a multichannel plate or channeltron was implemented.

\subsubsection{Rydberg electromagnetically-induced transparency}

In Fig. 8 we observe Rydberg EIT in the microscopic atom cloud. Here, the control light couples the intermediate state to the relatively low lying Rydberg state $30 S_{1 / 2}$ where van-der-Waals interactions do not play an important role. The sequence employed is the same as the absorption sequence in Fig. 4, except for the presence of 

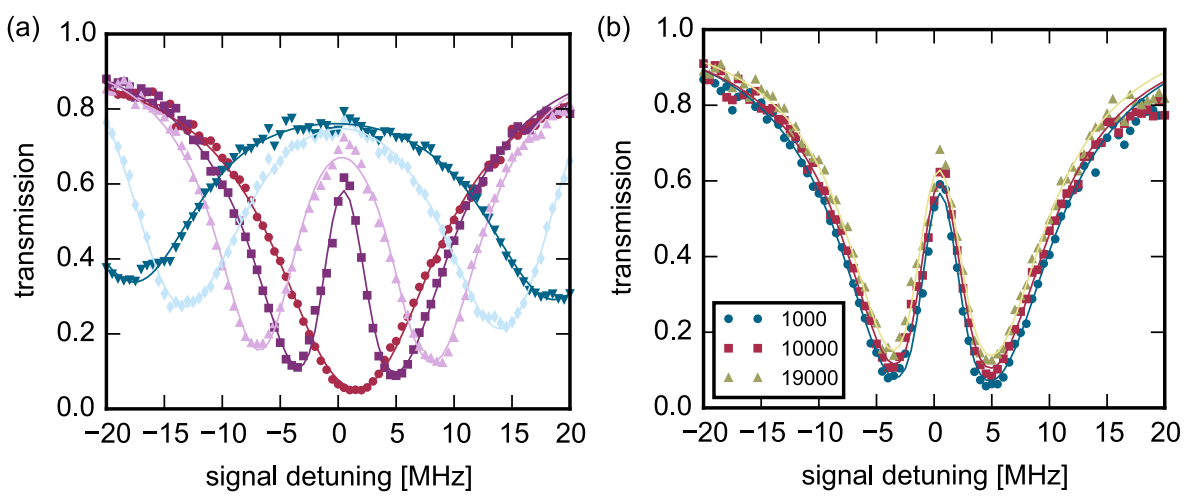

Fig. 8. Observation of Rydberg EIT at $30 S_{1 / 2}$. (a) EIT/Autler-Townes spectra for different control Rabi frequencies. From fits to the spectra we obtain in $\mathrm{MHz}, 7.9 \pm 0.1$ (purple squares), $14.6 \pm 0.1$ (pink triangles), $28.6 \pm 0.2$ (light blue diamonds), and $35.8 \pm 0.3$ (dark blue triangles). An absorption spectrum is shown for reference (red circles). (b) EIT spectra taken after 1000 (blue circles), 10000 (red squares), and 19000 (yellow triangles) repetitions on the same atom cloud. No significant reduction in optical depth is observed.

the control light during the entire $1 \mu$ s signal pulse which again contains 2.6 photons on average. In Fig. 8(a), signal transmission spectra are presented for different control intensities showing the transition from EIT to Autler-Townes splitting. The transmission spectra are fitted using

$$
T\left(\Delta_{S}\right)=\exp \left(\frac{-O D\left(\Gamma^{2}+\frac{\Gamma \gamma \Omega_{C}^{2} / 4}{\gamma^{2}+\left(\Delta_{S}-\Delta_{0}+\Delta_{C}\right)^{2}}\right)}{\left(\Gamma+\frac{\gamma \Omega_{C}^{2} / 4}{\gamma^{2}+\left(\Delta_{S}-\Delta_{0}+\Delta_{C}\right)^{2}}\right)^{2}+\left(\frac{\Omega_{C}^{2}\left(\Delta_{S}-\Delta_{0}+\Delta_{C}\right) / 4}{\gamma^{2}+\left(\Delta_{S}-\Delta_{0}+\Delta_{C}\right)^{2}}-\left(\Delta_{S}-\Delta_{0}\right)\right)^{2}}\right)
$$

where $O D, \Gamma, \gamma, \Omega_{C}, \Delta_{C}$ and $\Delta_{0}$ are free fitting parameters corresponding to the optical depth, the effective linewidth of the signal transition, the effective linewidth of the EIT, the control Rabi frequency, the control detuning and the offset of the absorption line.

By fitting the spectra we can extract the control Rabi frequencies $\Omega_{C} / 2 \pi=7.9$, 14.6, 28.6, and $35.8 \mathrm{MHz}$, respectively. We note that signal detuning $\Delta_{S} / 2 \pi$ for which the transmission is minimal in absence of the control beam, as well as the peaks of the EIT transmission windows are slightly shifted from $0 \mathrm{MHz}$ due to a Zeeman shift induced by the quantisation field, which is applied along the lens axis.

As Rydberg atoms in $n S_{1 / 2}$ are not trapped at a wavelength of $910 \mathrm{~nm}$, a potential mechanism for trap loss arises if any Rydberg population is present when the trap is switched back on. We therefore observe EIT spectra taken after a different number of shots performed on the same cold atom cloud, similar to the absorption experiment presented before. In Fig. 8(c) we present transmission spectra taken after 1000, 10000, and 19000 repetitions, again showing no significant reduction in optical depth.

\section{Photon storage and strong photon-interactions}

Below, we present a variety of experiments showing evidence of photon interactions mediated by Rydberg excitations: In EIT spectra, we observe a strong optical nonlinearity. Afterwards, we give a brief overview and demonstration of photon storage. For storage in Rydberg states with sufficiently high principal quantum number, 


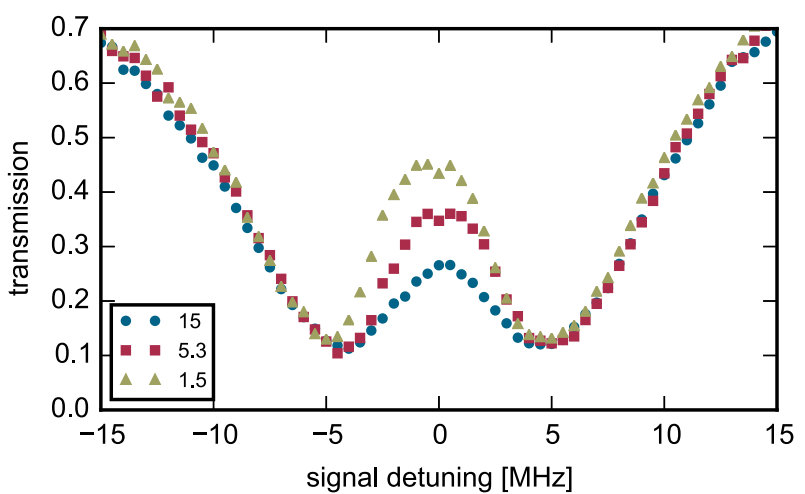

Fig. 9. Electromagnetically induced transparency at $80 S_{1 / 2}$. The spectra shown were taken for signal pulses with mean photon numbers of $15 \pm 1$ (blue circles), $5.3 \pm 0.4$ (red squares), and $1.5 \pm 0.1$ (yellow triangles). The transmission at resonance is suppressed as the intensity is increased due to the effective optical non-linearity as Rydberg interactions are mapped onto the light field.

we observe saturation as the mean incoming photon number is increased and obtain sub-Poissonian photon statistics indicating the retrieval of non-classical states of light. Finally, we perform microwave spectroscopy on photons on the transition between the $80 S_{1 / 2}$ state in which the photons are stored, and $79 P_{1 / 2}$ and observe broadening and a change of lineshape depending on the number of photons stored which can be explained by the introduction of resonant dipole-dipole interactions.

\subsection{Suppression of electromagnetically induced transparency}

The first observations of strong optical non-linearities induced by Rydberg interactions mapped onto light fields were made by observing a suppression of Rydberg EIT for increasing rates of incoming signal photons [8]. If the incident signal photon rate is low, photons are well separated in space and are not subjected to Rydberg induced interactions with other photons. In the vicinity of another photon, van-der-Waals interactions lead to a blockaded volume with blockade radius $R_{B}^{(6)}=\left(C_{6} / \hbar \Delta_{E I T}\right)^{1 / 6}$ where $\Delta_{E I T}$ corresponds to the width of the EIT transparency window. Inside the blockade, the Rydberg state is shifted out of resonance with the control field such that the signal light sees an effective two-level system and the blockaded volume is rendered opaque. If the rate of signal photons is high enough for photons to be propagating closer than $R_{B}^{(6)}$, the signal light starts to be absorbed and scattered. As the rate is further increased, more photons are propagating through the atomic cloud and the blockaded volume grows and suppression of the transparency feature is enhanced. This strongly non-linear response of the medium is shown in Fig. 9, where we present EIT spectra taken at $80 S_{1 / 2}$ for $1 \mu$ s signal pulses with mean photon numbers 14.7 , 5.3 , and 1.5. The control light has a Rabi frequency of $7.3 \pm 0.6 \mathrm{MHz}$. Each data point is averaged over 40000 shots.

The suppression of the transmission is much less pronounced than in other experiments $[11,20,21]$ working with larger and optically denser clouds. For now, this is no major disadvantage as we are mainly working with photon storage in microscopic ensembles since these provide well defined spatial localisation for the study of interaction between photons in closely spaced, yet spatially well distinguishable, channels. 


\subsection{Photon storage}

The transmission of resonant signal light in EIT is a consequence of a modified complex susceptibility of the three-level medium in the presence of the control field. Hence, not only the transmission, but also the dispersion and the group index of the medium are modified such that the Rabi frequency $\Omega_{C}$ of the control field can be used to control the group velocity of a signal photon and store it inside the medium. A convenient concept to explain the propagation and photon storage are dark state polaritons [58]. Here, a signal photon entering the atomic medium is considered to be in a superposition of a light field and an atomic excitation in the medium, commonly referred to as spin wave. The admixture of photonic and atomic character can be controlled by $\Omega_{C}$ and the atomic density: if $\Omega_{C}$ is large, the photonic character dominates and the group velocity is high, while for $\Omega_{C}=0$ the nature of the polariton is entirely atomic and the group velocity is reduced to zero. To store a signal photon as a Rydberg excitation, we reduce $\Omega_{C}$ to zero as it propagates in the medium. To retrieve the stored photon again, $\Omega_{C}$ is ramped to its initial value.

The retrieved light is preferentially emitted into the spatial mode of the incoming photon. This can be best explained by considering the stored photon in a collective superposition state of $N$ atoms:

$$
|R\rangle=\frac{1}{\sqrt{N}} \sum_{j=1}^{N} \mathrm{e}^{\mathrm{i}\left(\mathbf{k}_{\mathbf{C}}+\mathbf{k}_{\mathbf{S}}\right) \cdot \mathbf{r}_{\mathbf{j}}}\left|g_{1}, \ldots, r_{j}, \ldots, g_{N}\right\rangle
$$

where $\mathbf{r}_{\mathbf{j}}$ is the position of atom $j, \mathbf{k}_{\mathbf{S}}$ and $\mathbf{k}_{\mathbf{C}}$ are the wavevector of the light fields and $\left|g_{1}, \ldots, r_{j}, \ldots, g_{N}\right\rangle$ denotes a state with all atoms except $j$ in the ground, and $j$ in a Rydberg state $|r\rangle$. As the control field is switched back on, the system evolves from $|R\rangle$ to the state

$$
|E\rangle=\frac{1}{\sqrt{N}} \sum_{j=1}^{N} \mathrm{e}^{\mathrm{i} \mathbf{k}_{\mathbf{s}} \cdot \mathbf{r}_{\mathrm{j}}}\left|g_{1}, \ldots, e_{j}, \ldots, g_{N}\right\rangle
$$

where the previous Rydberg excitation has been transferred to the intermediate state $|e\rangle$. From here, the system decays into the collective ground state $|G\rangle$ through spontaneous emission of a signal photon. As the phase factor $\mathrm{e}^{\mathrm{i} \mathbf{k}_{\mathbf{s}} \cdot \mathbf{r}_{\mathbf{j}}}$ corresponds to the phase of the original signal photon, the retrieved light is preferentially emitted into the same spatial mode due to constructive interference of the emission of all $N$ atomic dipoles in the forward direction [59]. Here, sufficient optical depth is crucial to achieve good directionality of the retrieved light, as the interference effect is enhanced as $N$ increases. Due to the importance of the phase factors for the directionality, it is also affected by dephasing of the collective superposition, e.g. due to atomic motion, but also due to interactions of Rydberg excitations with each other [33,62].

\subsubsection{Demonstration of photon storage}

In Fig. 10 we show a typical photon storage and retrieval sequence. The timings are shown in Fig. 10(a). The photons are stored for $556 \mathrm{ns,}$, in the low-lying Rydberg state $30 S_{1 / 2}$. The incoming signal pulse has a mean photon number of $11 \pm 1$ and a duration of $350 \mathrm{~ns}$. The control light is switched on well before the signal pulse and turned off just before the end of the signal pulse. The Rabi frequency during the storage is $\Omega_{C} / 2 \pi=18 \pm 1 \mathrm{MHz}$. To retrieve the stored photons, the control light is switched back on at a Rabi frequency of $\Omega_{C} / 2 \pi=44 \pm 3 \mathrm{MHz}$ corresponding to the maximum 
(a)

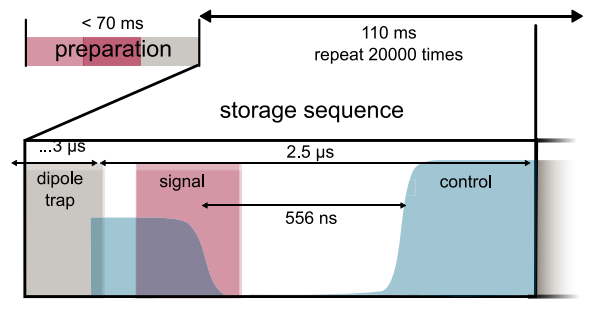

(b)

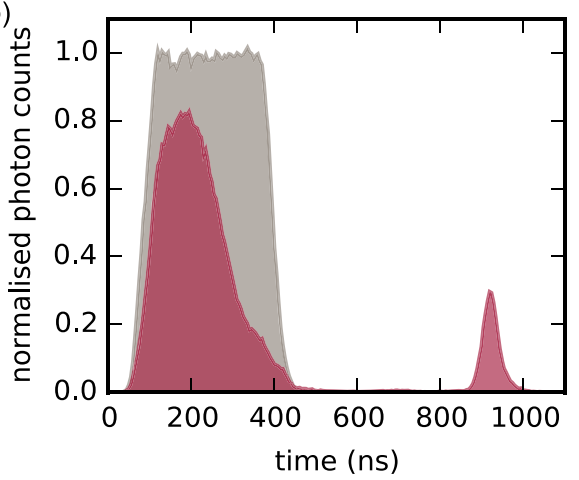

Fig. 10. Storage and retrieval of photons as Rydberg excitations at $30 S_{1 / 2}$. (a) Timing sequence. (b) Signal photons detected during storage and retrieval. Initially, the atom cloud is transparent. As $\Omega_{C}$ is reduced to zero, the absorption increases and photons are stored. Retrieval is observed after $556 \mathrm{~ns}$ of storage. The grey area corresponds to the incoming signal pulse in the absence of atoms. We note that a small amount of photon leakage is observed around $700 \mathrm{~ns}$ as a result of insufficient control light extinction.

control intensity available. The optical depth is on the order of $\approx 2.5$ and we estimate the total number of atoms to be on the order of a few $10^{3}$.

Figure 10(b) shows the detection of signal photons during the entirety of the sequence acquired over $2 \cdot 10^{7}$ repetitions. For reference, the grey area shows the profile of the incoming signal pulse in the absence of atoms, the red area shows the signal photons detected during the storage and retrieval sequence described above. At the beginning of the sequence, $\Omega_{C}$ is constant such that the signal light propagates through the atomic cloud under EIT conditions and most signal photons are transmitted. As $\Omega_{C}$ is turned to zero, the group velocity of the signal photons is reduced to zero, such that they are written into the medium as a spin wave and stored as a Rydberg excitation. As $\Omega_{C}$ is reduced, a decrease in the transmission can be observed. Once the control light is turned back on, we observe a pulse of retrieved signal photons. We note that the width of the retrieved pulse varies with $\Omega_{C}$. In this example, we chose $\Omega_{C}$ to be maximal in order to achieve the narrowest retrieval window possible. In general, the shape of the retrieved pulse is not only linked to the intensity, but also to the temporal profile of $\Omega_{C}$.

In the case of non-perfect extinction of the control light during the storage, the group velocity of the signal photons determined by $\Omega_{C}$ is non-zero, such that there is a slight probability to retrieve photons prematurely. To illustrate the consequences, the sequence shown in Fig. 10(b) was taken with insufficient extinction. The control intensity fluctuates during the storage sequence and as a consequence early retrieval (around $700 \mathrm{~ns}$ ) is observed. The fluctuations are caused by imperfect switching of the EOM due to the short transient time. To improve the extinction, the control intensity is monitored on a fast photodiode and we carefully adapt the control voltage profile applied to the EOM during the storage time, if necessary, before each set of experimental runs.

\subsubsection{Photon blockade}

A simple experiment to demonstrate mapping of the strong interactions between Rydberg atoms onto photons stored as Rydberg excitations is to measure the number of retrieved signal photons as function of the incoming photon number. This is in 


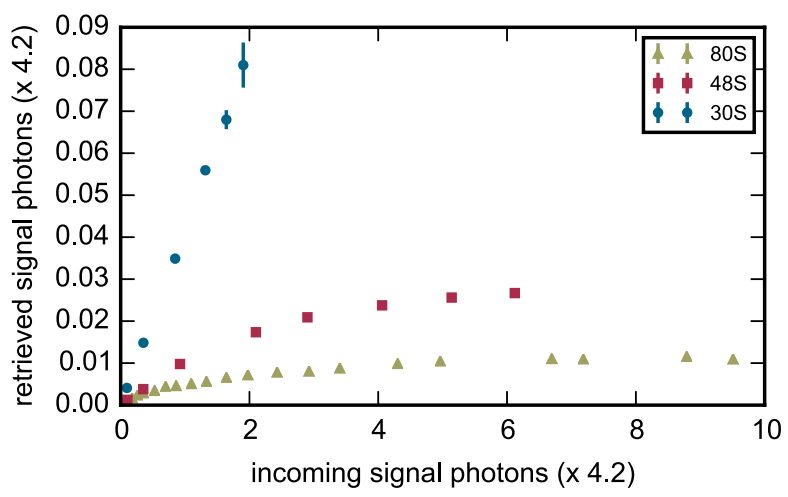

Fig. 11. Saturation of photon storage. For storage in $30 S_{1 / 2}$ (blue circles), a linear relation between the incoming and retrieved mean photon numbers is observed indicating the absence of interactions, while for $48 S_{1 / 2}$ (red squares) and $80 S_{1 / 2}$ (yellow triangles) the number of retrieved photon saturates giving evidence of the non-linearity arising as Rydberg blockade induced by van-der-Waals interactions limits the number of stored photons.

analogy to early experiments on Rydberg blockade [60,61], where the number of detected Rydberg atoms saturated at higher intensities of the excitation lasers. We measure the number of retrieved photons following $278 \mathrm{~ns}$ of storage as a function of the mean number of incoming signal photons in a coherent pulse of $350 \mathrm{~ns}$ duration $\left(400 \mathrm{~ns}\right.$ for $\left.80 S_{1 / 2}\right)$. The photons are stored in the states $30 S_{1 / 2}, 48 S_{1 / 2}$, and $80 S_{1 / 2}$ using control Rabi frequencies of $\Omega_{C} / 2 \pi=18 \pm 1 \mathrm{MHz}, 15.6 \pm 0.9 \mathrm{MHz}$, and $9.0 \pm$ $0.7 \mathrm{MHz}$, respectively. The results are presented in Fig. 11. For storage in $30 S_{1 / 2}$ a linear relation between the number of incoming and retrieved photons is observed. This behaviour at $n=30$ is expected as the number of Rydberg excitations needed to blockade the entire atomic cloud, exceeds the photon number of the incoming signal pulse. As $n$ is increased, the blockade radius increases as $R_{B}^{(6)} \propto n^{11 / 6}$ and the number of excitations needed to blockade the storage medium falls below the number of signal photons. Consequently, a non-linear relation and saturation of the retrieved number can be observed for storage in $48 S_{1 / 2}$ and $80 S_{1 / 2}$ giving evidence of the arising optical non-linearity and resulting effective photon blockade. We note that the statistical errorbars on most datapoints are too small to be visible thanks to the high number of repetitions $\left(2 \cdot 10^{6}\right.$ for $30 S_{1 / 2}, 4 \cdot 10^{6}$ for $48 S_{1 / 2}$, and $6 \cdot 10^{6}$ for $\left.80 S_{1 / 2}\right)$, the major contribution to the error in the photon numbers therefore arises from the systematic error in the detection efficiency.

\subsubsection{Photon statistics: non-classical light}

As the size of the microscopic trap used for photon storage in our experiment is of the same order as $R_{B}^{(6)}$, the effective photon blockade demonstrated above should impose a strong limitation on the number of stored photons to just a few. Hence, we expect that the effective photon blockade demonstrated above can be exploited to generate non-classical states of light from an incoming classical coherent pulse as demonstrated previously $[12,13]$. In the most extreme case where the entire atomic ensemble is blockaded by a single Rydberg excitation, a single photon source could be implemented [12].

In order to verify the quantum nature of the retrieved light, we analyse the photon statistics by performing a Hanbury-Brown Twiss experiment (see Fig. 7 for the 


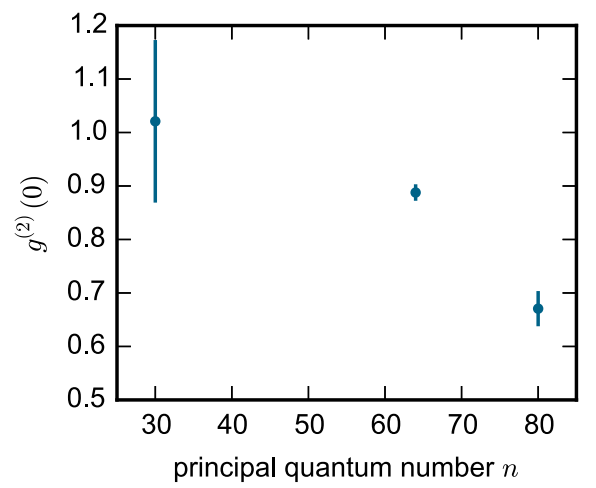

Fig. 12. Measurement of the second order correlation function $g^{(2)}(\tau=0)$ of photons retrieved after storage. The photons were stored in the Rydberg states $30 S_{1 / 2}, 64 S_{1 / 2}$, and $80 S_{1 / 2}$ for $278 \mathrm{~ns}$. For $64 S_{1 / 2}$, and $80 S_{1 / 2}$, we find $g^{(2)}(0)<1$ indicating sub-Poissonian counting statistic and the retrieval of non-classical states of light. The errorbars are estimated by calculating the standard error over multiple subsets of the data.

setup). The non-classicality can be verified by measuring the temporal second order correlation function $g^{(2)}(\tau=0)$ which is determined by comparing the probability of two photon coincidences at a time delay of $\tau=0$ to the probability of single photon counting events. For a classical coherent state with Poissonian photon number statistics $g^{(2)}(\tau=0)=1$, while for a quantum state of light with subPoissonian statistics $g^{(2)}(0)<1$ and in the most extreme case of a single photon Fock state $g^{(2)}(0)=0$.

Figure 12 shows the results for $g^{(2)}(0)$ for storage at Rydberg states $30 S_{1 / 2}$, $64 S_{1 / 2}$, and $80 S_{1 / 2}$. We store the photons again for a relatively short duration of $278 \mathrm{~ns}$, to minimise the influence of interaction induced [62] and motional dephasing. The incoming signal pulses are again $350 \mathrm{~ns}$ long and contain a mean of $9.5 \pm 0.4$ photons $\left(3.0 \pm 0.2\right.$ for $\left.30 S_{1 / 2}\right)$. The control Rabi frequencies are $\Omega_{C} / 2 \pi=19 \pm 1 \mathrm{MHz}$, $7.7 \pm 0.6 \mathrm{MHz}$, and $4.9 \pm 0.4 \mathrm{MHz}$ respectively for both storage and retrieval. At $30 S_{1 / 2}$ no suppression of $g^{(2)}(0)=1.0 \pm 0.2$ and therefore Poissonian photon statistics are observed as expected due to the lack of interactions and the incoming coherent signal pulse. In contrast, we obtain values of $g^{(2)}(0)=0.89 \pm 0.02$ at $64 S_{1 / 2}$ and $g^{(2)}(0)=0.67 \pm 0.03$ at $80 S_{1 / 2}$ indicating the retrieval of non-classical states of light. Each datapoint was taken over $3 \cdot 10^{7}$ storage and retrieval cycles.

In previous storage experiments, stronger suppression of $g^{(2)}(0)$ has been observed at comparable principal quantum numbers. We attribute this mainly to the use of a smaller excitation volume fully blockaded by fewer photons [12], and longer storage times [13], which lead to a reduction in the higher photon number components of the retrieved state as their read-out is suppressed due to interaction induced dephasing $[33,62]$.

\subsection{Microwave spectroscopy of stored photons}

Driving microwave transitions between nearby Rydberg states, e.g. from an initial $n S_{1 / 2}$ to an adjacent $(n-1) P$ state, can be used to induce resonant dipole-dipole interactions in a system of photons stored in $n S_{1 / 2}$ where initially only van-der-Waals interactions are present [13,63]. Microwave spectroscopy provides a tool to probe the interactions [64-66]. By detecting the retrieved light after a storage process, our 
(a)

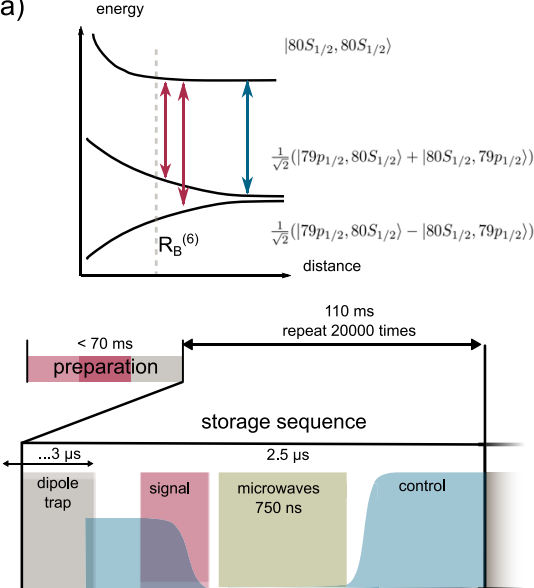

(b)

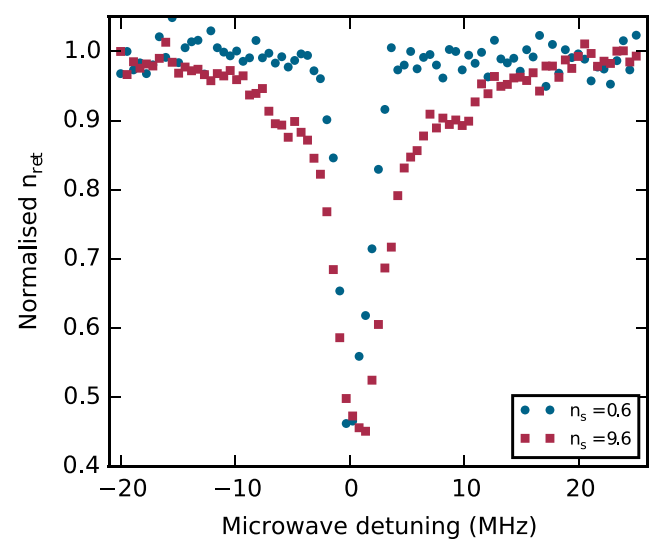

Fig. 13. Microwave spectroscopy on photons stored as Rydberg excitations on the $80 S_{1 / 2}$ to $79 P_{1 / 2}$ transition. (a) Level scheme sketching the distance dependence of pair state interaction energies relevant for the resonant dipole interaction induced by the microwave coupling and experimental sequence. (b) Normalised photon retrieval from $80 S_{1 / 2}$ vs. microwave detuning for incoming signal pulses with mean photon numbers 0.6 and 9.6. The broadening observed for $n_{S}=9.6$, agrees with the interaction induced energy shifts for excitation pairs separated by $R_{B}^{(6)}\left(80 S_{1 / 2}\right) \approx 10 \mu \mathrm{m}$ (see red arrows in part (a) and text).

setup allows an optical read-out of the Rydberg excitations present in the cloud. Since the control field is resonant with a single $n S_{1 / 2}$ state, this detection method is state selective and well suited to study interaction processes involving multiple Rydberg states.

To demonstrate this, we use a microwave field to probe the interaction energy of pairs (or clusters) of Rydberg excitations in states $80 S_{1 / 2}$ and $79 P_{1 / 2}$ (see Fig. 13(a)). We start by storing signal photons as $80 S_{1 / 2}$ Rydberg excitations. Following the storage process, we apply a microwave pulse which is scanned around the $80 S_{1 / 2} \leftrightarrow$ $79 P_{1 / 2}$ transition which we find at $7.704 \mathrm{GHz}$. After $1 \mu \mathrm{s}$ we retrieve the photons from the $80 S_{1 / 2}$ state. Around the resonance frequency we observe a reduction in the retrieval rate since Rydberg excitations in $79 P_{1 / 2}$ are not read out. The results are shown in Fig. 13(b). We present scans for two different mean photon numbers $n_{s}$. At $n_{s}=0.60 \pm 0.05$ where in the majority of instances only a single excitation is created, and at $n_{s}=9.6 \pm 0.8$ where multiple excitations can be present. If only one excitation is present, excitation of $79 P_{1 / 2}$ Rydberg atoms occurs only at resonance, whereas for $n_{s}=9.6$, we observe transfer to $79 P_{1 / 2}$ for a broader window around the resonance, giving evidence of the resonant dipole-dipole interactions introduced into the system. We evaluate the interaction energies to show that our observation is compatible with probing a fully blockaded Rydberg gas. The $C_{6}$ coefficient for $80 S_{1 / 2}$ is $4.25 \mathrm{THz} / \mu \mathrm{m}^{6}$ which combined with a power-broadened excitation linewidth of $\Omega_{C} / 2 \pi=4.4 \mathrm{MHz}$ implies a van-der-Waals blockade radius of around $R_{B}^{(6)}=10 \mu \mathrm{m}$. The $C_{3}$ coefficient for the interaction between $80 S_{1 / 2}$ and $79 P_{1 / 2}$ is $8.36 \mathrm{GHz} / \mu \mathrm{m}^{3}$, yielding an interaction energy of $8.4 \mathrm{MHz}$ for a separation of $10 \mu \mathrm{m}$, which roughly corresponds to the observed broadening (see Fig. 13(a)). We note that this technique may be suited to probe spatial correlations [67] between photons stored as Rydberg excitation emerging in a saturated, quasi 1D microscopic dipole trap [63], though further investigation is beyond the scope of this paper. 


\section{Outlook and conclusion}

The implementation of the experimental setup described here paves the way for interesting experiments in quantum non-linear optics with Rydberg atoms. In particular, the fast repetition rate achieved allows the collection of large datasets in short timescales - most datasets presented were taken in a matter of minutes. This should, for example, enable us to study the dynamics of photon storage and retrieval in much more detail compared to previous experiments [33], and allow analysing the photon counting statistics in narrow time intervals within the retrieved signal pulse.

The realisation of two adjacent microscopic cold atom clouds provides a good starting point to study non-local interactions between individual photons [68] in two spatially separated optical modes, in the spirit of similar experiments with closely spaced single atoms in optical tweezers $[69,70]$. Besides applications in quantum non-linear optics, the system should be suited to study spin exchange [55] and resonant energy transfer $[18,71,72]$ if resonant dipole interactions are introduced by partly transferring Rydberg excitations to a $n P$ state using the in-vacuum microwave antennae.

A limitation for technical applications remains the low storage efficiency. The current efficiency record for ground state storage in a cold atomic medium stands at 0.78 [73], while for Rydberg states a value of 0.2 has been reported recently [22]. In both cases, optical depth and volume of the atom clouds are larger than in our setup. To study interactions between photons stored in the side-by-side traps at a well defined distance, we ideally want the atoms to be tightly confined in the lens axis direction. This could be achieved by adding a crossed trapping beam with a waist on the order of $20 \mu \mathrm{m}$ to compress the clouds longitudinally. At the same time this beam could serve as a reservoir to transfer a larger fraction atoms from the MOT into the microscopic traps. Another viable approach to create denser, more tightly confined ensembles would be the combination of two microscopic traps using co-propagating beams with different waists [74]. At high densities however, care must be taken though as interactions between Rydberg and ground state atoms can introduce an additional dephasing mechanism $[19,75,76]$. Another approach could exploit collectively enhanced optical cross sections of atoms arranged in a 2D array [77].

Besides quantum non-linear optics, the detection of microwave fields using EIT is another field of interest [28]. In comparison to room-temperature vapour cells, cold atom experiments offer the advantage of narrower linewidths thus making it easier to detect Autler-Townes splitting caused by extremely weak microwave fields. However, the sensitivity generally suffers in comparison due to the time it takes to prepare a cold atom cloud. Thanks to the high repetition rate achieved however, this overhead could be significantly reduced.

In summary, we have implemented an experimental apparatus well suited for quantum non-linear optics with Rydberg atoms that operates at a very high effective repetition rate of $110 \mathrm{kHz}$. We store photons as Rydberg excitations in a microscopic cold atom cloud and observe evidence of strong effective photon interactions in the form of saturation of the retrieved photon number. Analysis of the photon statistics reveals the generation of non-classical states of light. Performing microwave spectroscopy on photons stored as Rydberg excitations, we observe a photon number dependent change in the line shape which may provide information on the spatial distribution of Rydberg excitations in future experiments. Finally, the realisation of two side-byside microscopic traps opens up perspectives to study non-local interactions between individual photons propagating in spatially separated modes.

We thank the past and present members of the Durham Rydberg Quantum Optics team for their countless contributions to this work: C.S. Adams and M.P.A. Jones for supervision, 
invaluable guidance, and constant support, T. Ilieva for assistance carrying out the experiments, D.J. Szwer for implementing the ns-data acquisition and experimental control, as well as D. Paredes, K.J. Weatherill, and D. Maxwell for their contributions in the planning stage of the apparatus. We thank all members of the ITN "COHERENCE" for fruitful exchange over the last years, especially the group of A. Browaeys for collaboration on the lens design and the group of M. Weidemüller for collaboration on the 2D MOT. In the Durham Physics Department, we thank the group of S.L. Cornish for sharing their experimental control software, A. Hindmarch for applying the platinum coatings, and the mechanical workshop team for outstanding support. This work was supported financially by the European Union through the Marie Curie ITN "COHERENCE", FET-PROACT project "RySQ", and a Marie Curie Individual Fellowship to P.H., by EPSRC, DSTL, and Durham University.

The data presented in this paper are available at http://dx.doi.org/10.15128/ r1fq977t77z.

\section{References}

1. J.D. Pritchard, K.J. Weatherill, C.S. Adams, Ann. Rev. Cold Atoms Mol. 1, 301 (2013)

2. T.F. Gallagher, Rydberg Atoms (Cambridge University Press, Cambridge, 1994)

3. M. Saffman, T.G. Walker, K. Mølmer, Rev. Mod. Phys. 82, 2313 (2010)

4. M. Fleischhauer, A. Imamoglu, J.P. Marangos, Rev. Mod. Phys. 77, 633 (2005)

5. A.K. Mohapatra, T.R. Jackson, C.S. Adams, Phys. Rev. Lett. 98, 113003 (2007)

6. M.D. Lukin, M. Fleischhauer, R. Cote, L.M. Duan, D. Jaksch, J.I. Cirac, P. Zoller, Phys. Rev. Lett. 87, 037901 (2001)

7. D. Comparat, P. Pillet, J. Opt. Soc. Am. B 27, A208 (2010)

8. J.D. Pritchard, D. Maxwell, A. Gauguet, K.J. Weatherill, M.P.A. Jones, C.S. Adams, Phys. Rev. Lett. 105, 193603 (2010)

9. V. Parigi, E. Bimbard, J. Stanojevic, A.J. Hilliard, F. Nogrette, R. Tualle-Brouri, A. Ourjoumtsev, P. Grangier, Phys. Rev. Lett. 109, 233602 (2012)

10. A.V. Gorshkov, J. Otterbach, M. Fleischhauer, T. Pohl, M.D. Lukin, Phys. Rev. Lett. 107, $133602(2011)$

11. T. Peyronel, O. Firstenberg, Q.-Y. Liang, S. Hofferberth, A.V. Gorshkov, T. Pohl, M.D. Lukin, V. Vuletić, Nature 488, 57 (2012)

12. Y.O. Dudin, A. Kuzmich, Science 336, 887 (2012)

13. D. Maxwell, D.J. Szwer, D. Paredes-Barato, H. Busche, J.D. Pritchard, A. Gauguet, K.J. Weatherill, M.P.A. Jones, C.S. Adams, Phys. Rev. Lett. 110, 103001 (2013)

14. C.S. Hofmann, G. Günter, H. Schempp, M. Robert-de-Saint-Vincent, M. Gärttner, J. Evers, S. Whitlock, M. Weidemüller, Phys. Rev. Lett. 110, 203601 (2013)

15. O. Firstenberg, T. Peyronel, Q.-Y. Liang, A.V. Gorshkov, M.D. Lukin, V. Vuletić, Nature 502, 71 (2013)

16. B. Olmos, W. Li, S. Hofferberth, I. Lesanovsky, Phys. Rev. A 84, 041607 (2011)

17. G. Günter, M. Robert-de-Saint-Vincent, H. Schempp, C.S. Hofmann, S. Whitlock, M. Weidemüller, Phys. Rev. Lett 108, 013002 (2012)

18. G. Günter, H. Schempp, M. Robert-de-Saint-Vincent, V. Gavryusev, S. Helmrich, C.S. Hofmann, S. Whitlock, M. Weidemüller, Science 342, 954 (2013)

19. S. Baur, D. Tiarks, G. Rempe, S. Dürr, Phys. Rev. Lett. 112, 073901 (2014)

20. H. Gorniaczyk, C. Tresp, J. Schmidt, H. Fedder, S. Hofferberth, Phys. Rev. Lett. 113, $053601(2014)$

21. D. Tiarks, S. Baur, K. Schneider, S. Dürr, G. Rempe, Phys. Rev. Lett. 113, 053602 (2014)

22. D. Tiarks, S. Schmidt, G. Rempe, S. Dürr, Sci. Adv. 2, 160036 (2016)

23. A.K. Mohapatra, M.G. Bason, B. Butscher, K.J. Weatherill, C.S. Adams, Nat. Phys. 4, $890(2008)$

24. A. Tauschinsky, R.M.T. Thijssen, S. Whitlock, H.B. van Linden van den Heuvell, R.J.C. Spreeuw, Phys. Rev. A 81063411 (2010)

25. R.P. Abel, C. Carr, U. Krohn, C.S. Adams, Phys. Rev. A 84, 023408 (2011) 
26. H. Hattermann, M. Mack, F. Karlewski, F. Jessen, D. Cano, J. Fortágh, Phys. Rev. A 86, $022511(2012)$

27. K.S. Chan, M. Siercke, C. Hufnagel, R. Dumke, Phys. Rev. Lett. 112, 026101 (2014)

28. J.A. Sedlacek, A. Schwettmann, H. Kübler, R. Löw, T. Pfau, J.P. Shaffer, Nat. Phys. 8, $819824(2012)$

29. J.A. Sedlacek, A. Schwettmann, H. Kübler, J.P. Shaffer, Phys. Rev. Lett. 111, 063001 (2013)

30. J.A. Gordon, C.L. Holloway, A. Schwarzkopf, D.A. Anderson, S. Miller, N. Thaicharoen, G. Raithel, Appl. Phys. Lett. 105, 024104 (2014)

31. H.Q. Fan, S. Kumar, R. Daschner, H. Kübler, J.P. Shaffer, Opt. Lett. 39, 3030 (2014)

32. C.L. Holloway, J.A. Gordon, A. Schwarzkopf, D.A. Anderson, S.A. Miller, N. Thaicharoen, G. Raithel, Appl. Phys. Lett. 104, 244102 (2014)

33. D. Maxwell, D.J. Szwer, D. Paredes-Barato, H. Busche, J.D. Pritchard, A. Gauguet, M.P.A. Jones, C.S. Adams, Phys. Rev. A 89, 043827 (2014)

34. H. Gorniaczyk, C. Tresp, P. Bienias, A. Paris-Mandoki, W. Li, I. Mirgorodskiy, H.P. Büchler, I. Lesanovsky, S. Hofferberth, Nat. Commun. 7, 12480 (2016)

35. D. Paredes-Barato, C.S. Adams, Phys. Rev. Lett. 112, 040501 (2014)

36. L. Isenhower, E. Urban, X.L. Zhang, A.T. Gill, T. Henage, T.A. Johnson, T.G. Walker, M. Saffman, Phys. Rev. Lett. 104, 010503 (2010)

37. C. Ates, I. Lesanovsky, C.S. Adams, K.J. Weatherill, Phys. Rev. Lett. 110, 213003 (2013)

38. M. Ebert, A. Gill, M. Gibbons, X. Zhang, M. Saffman, T.G. Walker, Phys. Rev. Lett. 112, $043602(2014)$

39. E. Brion, K. Mølmer, M. Saffman, Phys. Rev. Lett. 99, 260501 (2007)

40. M. Ebert, M. Kwon, T.G. Walker, M. Saffman, Phys. Rev. Lett. 115, 093601 (2015)

41. K. Dieckmann, R.J.C. Spreeuw, M. Weidemüller, J.T.M. Walraven, Phys. Rev. A 58, 3891 (1998)

42. C.S. Hofmann, G. Günter, H. Schempp, N.M.L. Müller, A. Faber, H. Busche, M. Robert-de-Saint-Vincent, S. Whitlock, M. Weidemüller, Front. Phys. 9, 571 (2014)

43. S. Chaudhuri, S. Roy, C.S. Unnikrishnan, Phys. Rev. A 74, 023406 (2006)

44. T.G. Tiecke, S.D. Gensemer, A. Ludewig, J.T.M. Walraven, Phys. Rev. A 80, 013409 (2009)

45. J. Schoser, A. Batär, R. Löw, V. Schweikhard, A. Grabowski, Y.B. Ovchinnikov, T. Pfau, Phys. Rev. A 66, 023410 (2002)

46. R. Grimm, Y.B. Ovchinnikov, M. Weidemüller, Adv. At. Mol. Opt. Phys. 42, 95 (2000)

47. S. Zhang, F. Robicheaux, M. Saffman, Phys. Rev. A 84, 043408 (2011)

48. L. Li, Y.O. Dudin, A. Kuzmich, Nature 498, 466 (2013)

49. E.A. Goldschmidt, D.G. Norris, S.B. Koller, R. Wyllie, R.C. Brown, J.V. Porto, U.I. Safronova, M.S. Safronova, Phys. Rev. A 91, 032518 (2015)

50. G. Li, S. Zhang, L. Isenhower, K. Maller, M. Saffman, Opt. Lett. 37, 851 (2012)

51. A.L. Gaunt, T.F. Schmidutz, I. Gotlibovych, R.P. Smith, Z. Hadzibabic, Phys. Rev. Lett. 110, 200406 (2013)

52. F. Nogrette, H. Labuhn, S. Ravets, D. Barredo, L. Béguin, A. Vernier, T. Lahaye, A. Browaeys, Phys. Rev. X 4, 021034 (2014)

53. M.J. Piotrowicz, M. Lichtman, K. Maller, G. Li, S. Zhang, L. Isenhower, M. Saffman, Phys. Rev. A 88, 013420 (2013)

54. R. Löw, H. Weimer, J. Nipper, J.B. Balewski, B. Butscher, H.P. Büchler, T. Pfau, J. Phys. B 45, 113001 (2012)

55. D. Barredo, H. Labuhn, S. Ravets, T. Lahaye, A. Browaeys, C.S. Adams, Phys. Rev. Lett. 114, $113002(2015)$

56. D.J. McCarron, S.A. King, S.L. Cornish, Meas. Sci. Technol. 19, 105601 (2008)

57. R.P. Abel, A.K. Mohapatra, M.G. Bason, J.D. Pritchard, K.J. Weatherill, U. Raitzsch, C.S. Adams, Appl. Phys. Lett. 94, 071101 (2009)

58. M. Fleischhauer, M.D. Lukin, Phys. Rev. Lett. 84, 5094 (2000)

59. M.O. Scully, E.S. Fry, C.H.R. Ooi, K. Wodkiewicz, Phys. Rev. Lett. 96, 010501 (2006) 
60. D. Tong, S.M. Farooqi, J. Stanojevic, S. Krishnan, Y.P. Zhang, R. Côté, E.E. Eyler, P.L. Gould, Phys. Rev. Lett. 93, 063001 (2004)

61. K. Singer, M. Reetz-Lamour, T. Amthor, L.G. Marcassa, M. Weidemüller, Phys. Rev. Lett. 93, 163001 (2004)

62. F. Bariani, Y.O. Dudin, T.A.B. Kennedy, A. Kuzmich, Phys. Rev. Lett. 108, 030501 (2012)

63. S. Bettelli, D. Maxwell, T. Fernholz, C.S. Adams, I. Lesanovsky, C. Ates, Phys. Rev. A 88, $043436(2013)$

64. K. Afrousheh, P. Bohlouli-Zanjani, D. Vagale, A. Mugford, M. Fedorov, J.D.D. Martin, Phys. Rev. Lett. 93, 233001 (2004)

65. A. Reinhard, K.C. Younge, T. Cubel Liebisch, B. Knuffman, P.R. Berman, G. Raithel, Phys. Rev. Lett. 100, 233201 (2008)

66. R. Celistrino Teixeira, C. Hermann-Avigliano, T.L. Nguyen, T. Cantat-Moltrecht, J.M. Raimond, S. Haroche, S. Gleyzes, M. Brune, Phys. Rev. Lett. 115, 013001 (2015)

67. P. Schauß, M. Cheneau, M. Endres, T. Fukuhara, S. Hild, A. Omran, T. Pohl, C. Gross, S. Kuhr, I. Bloch, Nature 491, 87 (2012)

68. S. Sevinçli, N. Henkel, C. Ates, T. Pohl, Phys. Rev. Lett. 107, 153001 (2011)

69. E. Urban, T.A. Johnson, T. Henage, L. Isenhower, D.D. Yavuz, T.G. Walker, M. Saffman, Nat. Phys. 5, 110 (2009)

70. A. Gaëtan, Y. Miroshnychenko, T. Wilk, A. Chotia, M. Viteau, D. Comparat, P. Pillet, A. Browaeys, P. Grangier, Nat. Phys. 5, 115 (2009)

71. C.S.E. van Ditzhuijzen, A.F. Koenderink, J.V. Hernández, F. Robicheaux, L.D. Noordam, H.B. van Linden van den Heuvell, Phys. Rev. Lett. 100, 243201 (2008)

72. S. Ravets, H. Labuhn, D. Barredo, L. Béguin, T. Lahaye, A. Browaeys, Nat. Phys. 10, $914(2014)$

73. Y.-H. Chen, M.-J. Lee, I-C. Wang, S. Du, Y.-F. Chen, Y.-C. Chen, I.A. Yu, Phys. Rev. Lett. 110, 083601 (2013)

74. R. Bourgain, J. Pellegrino, A. Fuhrmanek, Y.R.P. Sortais, A. Browaeys, Phys. Rev. A 88, $023428(2013)$

75. J.B. Balewski, A.T. Krupp, A. Gaj, D. Peter, H.P. Büchler, R. Löw, S. Hofferberth, T. Pfau, Nature 502, 664 (2013)

76. A. Gaj, A.T. Krupp, J.B. Balewski, R. Löw, S. Hofferberth, T. Pfau, Nat. Commun. 5, $4546(2014)$

77. R.J. Bettles, S.A. Gardiner, C.S. Adams, Phys. Rev. Lett. 116, 103602 (2016)

Open Access This is an Open Access article distributed under the terms of the Creative Commons Attribution License (http://creativecommons.org/licenses/by/4.0), which permits unrestricted use, distribution, and reproduction in any medium, provided the original work is properly cited. 